This item was submitted to Loughborough's Research Repository by the author.

Items in Figshare are protected by copyright, with all rights reserved, unless otherwise indicated.

\title{
A feasibility study of the effect of phone-based feedback of other commuters' subjective experiences on driver intentions to change
}

PLEASE CITE THE PUBLISHED VERSION

http://dx.doi.org/10.1007/978-3-319-20886-2 51

\section{PUBLISHER}

(C) Springer

VERSION

AM (Accepted Manuscript)

\section{PUBLISHER STATEMENT}

This work is made available according to the conditions of the Creative Commons Attribution-NonCommercialNoDerivatives 4.0 International (CC BY-NC-ND 4.0) licence. Full details of this licence are available at: https://creativecommons.org/licenses/by-nc-nd/4.0/

\section{LICENCE}

CC BY-NC-ND 4.0

\section{REPOSITORY RECORD}

Ross, Tracy, Andrea Burris, Luis C.R. Oliveira, Bronia Arnott, and Vera Araujo-Soares. 2019. "A Feasibility Study of the Effect of Phone-based Feedback of Other Commuters' Subjective Experiences on Driver Intentions to Change". figshare. https://hdl.handle.net/2134/19015. 


\title{
A feasibility study of the effect of phone-based feedback of other commuters' subjective experiences on driver intentions to change
}

\author{
Tracy Ross ${ }^{1}$, Andrea Burris ${ }^{1}$, Luis Oliveira ${ }^{1}$, Bronia Arnott ${ }^{2}$, and Vera Araujo-Soares ${ }^{2}$ \\ ${ }^{1}$ Loughborough University, Loughborough, UK. \\ \{t.ross, a.n.burris, 1.oliveira\}@lboro.ac.uk \\ ${ }^{2}$ Newcastle University, Newcastle, UK. \\ \{bronia.arnott, vera.araujo-soares\}@newcastle.ac.uk
}

\begin{abstract}
Encouraging people to make sustainable transport choices remains a global challenge and many interventions have been attempted. This study investigated the reflection on own/others' subjective experiences (SE) as an intervention using a smartphone application as the intervention tool. Participants were car drivers and used the app to automatically capture and reflect on their commute journeys and experiences. The experimental group were also able to reflect upon others' experiences across car, walk and cycle modes. Others' experiences were designed based on a previous self-report study. Results of the study showed that quantitative measures of intentions to change were not affected by the intervention but that qualitative data showed that the positive experience of the active transport modes did bring about reflection on behaviour and a potential influence on opinions and intentions which warrants further study.
\end{abstract}

Keywords: Behaviour change $\cdot$ sustainable transport $\cdot$ active travel $\cdot$ own subjective experience $\cdot$ others' subjective experience $\cdot$ opinions $\cdot$ outcome expectations · intention to change $\cdot$ reflection $\cdot$ persuasion $\cdot$ Socio Cognitive Theory . HCI $\cdot$ mobile.

\section{Introduction}

A growing body of research investigates the use of self-tracking devices to influence behaviour in a range of activities. The application of technology can support the achievement of goals such as physical activity or increase self-understanding, which can lead to behaviour change [3]. With more wearable technologies being developed, there are more possibilities available for research based on persuasive strategies [7, 10]. Persuasive technology and methods can also be used to promote more sustainable travel behaviours [13].

Several studies have been conducted to test participants' responses to feedback received on personalised collected data on their own travel behaviours and choices, particularly mode use. The measures reported back to subjects often include reports, 
per mode, of quantifiable aspects such as cost, calories consumed or $\mathrm{CO}^{2}$ produced [8].

A growing body of research is investigating the relation between subjectively experienced well-being and transport $[4,6]$. There is some evidence that the way we feel can be influenced by the modes of transport we choose and our individual travel behaviours. Usually, people walking and cycling present higher levels of subjective wellbeing (SW) than car drivers and users of public transport [9, 11, 12]. Some of the explanations include individual beliefs that active commute provides further benefits (outcome expectations) such as improved health from desirable physical exercise, harmony with environmental values, lower costs, less stress and feelings of autonomy [1].

Past studies have captured subjective wellbeing through post-hoc recall and at a generic level, i.e. across all journeys of a specific type (e.g. walking). This study was part of a feasibility project which had an overall aim of investigating whether reflection on experiences captured in-situ, immediately after each journey, could encourage a shift to more sustainable modes (or at least a change of opinion or behavioural intentions). This study in particular, focused on designing an interface to capture and present Subjective Experience (SE) which comprised subjective well-being and subjective outcome experiences and investigating how participants perceived that information alongside the more usual measures such as calorie expenditure and carbon dioxide footprint (CO2). The hypothesis was that, reflecting on the more positive experiences of active transport mode users, would cause a shift in car driver opinions or intentions to change.

\section{Method}

This feasibility study used a randomised controlled before and after design with 18 participants, 9 allocated to the control condition and 9 to the experimental condition. All participants used a smartphone application which logged their journeys automatically and allowed them to manually capture and review their Subjective Experience (SE) (comprised of subjective well-being as a 'smiley face' rating and subjective outcome experience as a free-text comment) on its completion (over a 2 week period in late autumn/early winter). Participants in the experimental condition received an additional behaviour change technique; feedback on other commuters' subjective experience. The participants first received a briefing on the use of the app and completed a pre- intervention questionnaire in which they indicated, using a 5-point Likert scale, their intention to change their mode of transport in the next month. They then used the app. for 2 weeks (with a contact email half way through to check everything was proceeding well). During the 2 week trial statistics were collected on the number of views of the test screens (i.e. 'Capture SE', 'Own SE', 'Others' SE' screens). The final assessment stage was a face-to-face meeting where participants began by completing a post- intervention questionnaire comprising of the same questions as in the briefing. They then completed an interview which was semi-structured, comprising of questions on their opinions and travel behaviours, their intentions to change in the 
future, and it also provided the opportunity to go through each screen of the app, participants were asked to indicate using a 7-point scale how easy to understand and informative they found each screen.

\subsection{Smartphone application intervention}

An existing smartphone application (app) was used as a basis for interface development in this study. The app was called CarbonDiem and was produced by TravelAi. This app was purposefully selected because it had the power to automatically detect transport mode, thus reducing participant burden. The app uses data from sensors on the phone, such as the accelerometer and GPS to achieve this. It is important to note that this app was originally developed as one element of a carbon measurement service for companies and not as an app for individual reflection. As this original intent was not communicated to the participants as part of this study, this may have influenced the participants' responses to these features, which should be taken into account when considering the results section. The app developers, in a spirit of openness, kindly allowed us to share feedback on their screens in this paper nevertheless. These screens accounted for the 'auto-detected behaviour' shown in Figure 1.

The additional screens designed for the study were to (a) to capture and present one's own subjective experience ('self-reported' aspects shown in Figure 1) and (b) to enable reflection of others' subjective experience ('researcher-created data' shown in Figure 1).

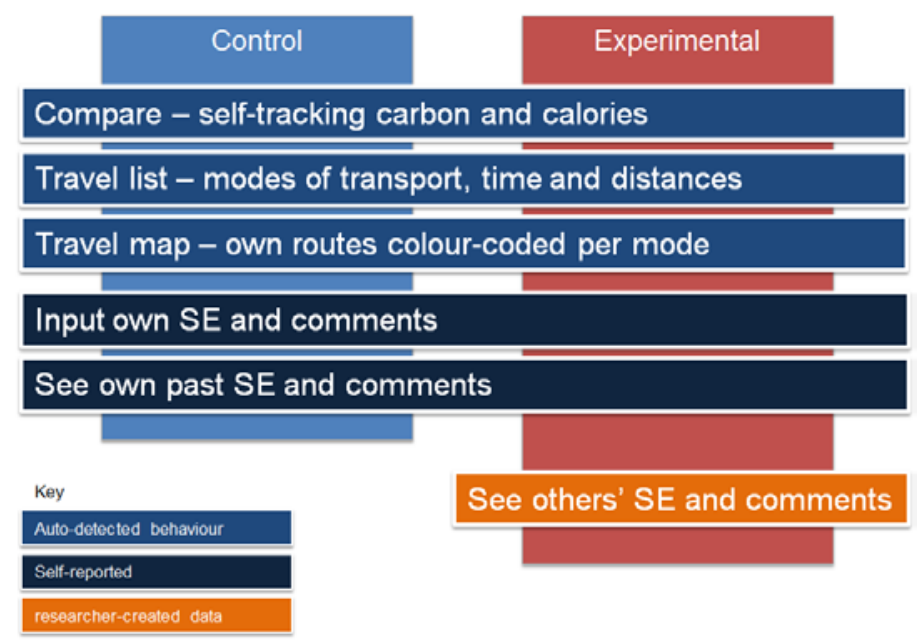

Fig. 1. Study Design 


\subsection{Interface design}

The first four screens (Figures 2 and 3) were part of the original app. The second three screens (Figures 4 and 5) are those designed for this study. The titles used for the figures relate to the coded boxes in Figure 1.
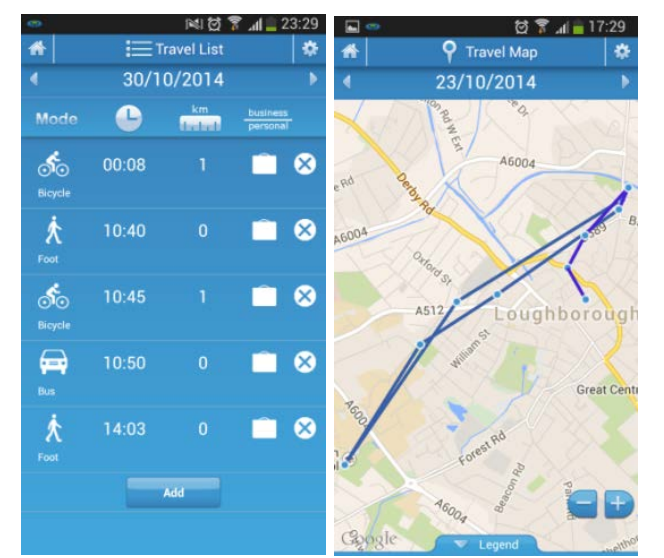

- Travel List with users' journeys each day. Includes mode, time, distance and purpose

- Travel Map of each journey (colour coded by mode)

- Modes available: Bicycle, Foot, Rail, Metro, Boat, Plane, Road (bus, car, motorbike, taxi)

- Data logged and available for the last 7 days.

Fig. 2. Travel List and Travel Map (original app screens)
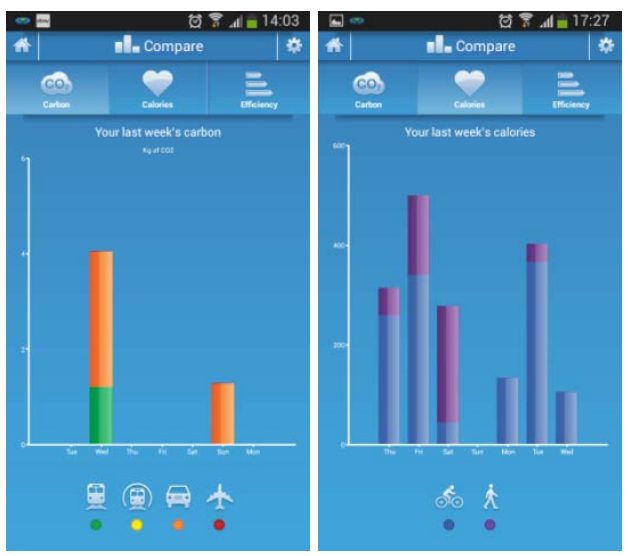

- Under the 'compare' icon users can see (i) 'Carbon', a graph of $\mathrm{KG} \mathrm{CO}^{2}$ per day per non-active mode (left hand screen), (ii). 'Calories', a graph of calories used per day per active mode (right hand screen) and (ii).'Efficiency', a graph of $\mathrm{Km}$ vs Kg of Co2, per day (not shown).

- All could be viewed by week or by month.

Fig. 3. Compare (original app screens) 

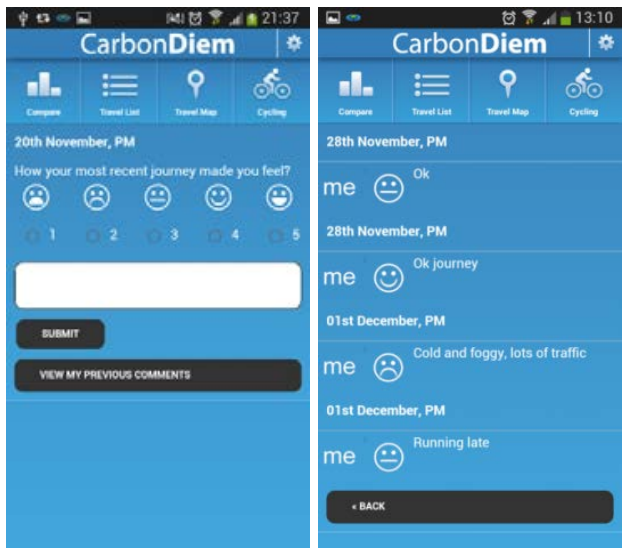

- Capture own SE: comprised of 'subjective wellbeing' rating and 'subjective outcome expectation' comments (left hand screen)

- Input by the user manually, entering a rating (e.g. smiley face) and free text about how they felt on their most recent commute

- Display own SE ratings and comments (right hand screen).

- Data logged and available for the last 7 days

Fig. 4. Input/see own SE ratings and comments (study screens)
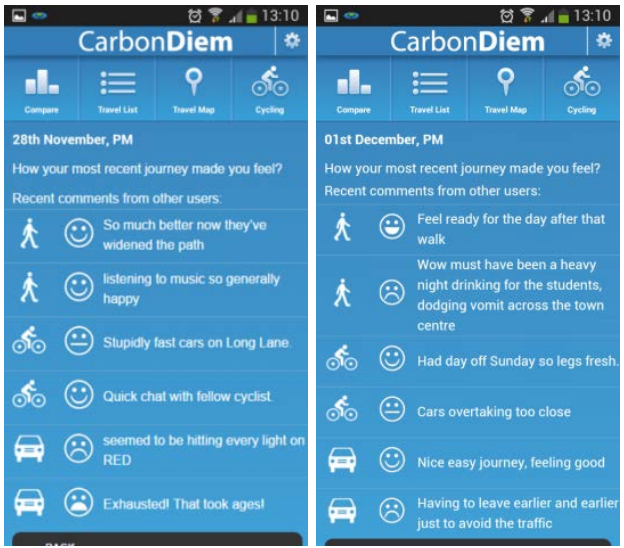

- Users saw others' SE by mode (changed twice per day to show past 12 hours), car, bike, walk only

- 2 quotes per each of 3 modes

- The smiley face ratings and quotes were created by the research team, based on a previous study i.e. they were not realtime travellers data (see further details below in section 2.3).

- This data was provided automatically once users submitted their SE data (but could be explored at any time)

Fig. 5. See others' SE ratings and comments (experimental only study screen)

\subsection{Others' subjective experiences}

Although 'Own SE' was obviously genuine, real-time information, the 'Others' SE' smiley faces and comments were created by the research team based on data collected in a previous study by the research team. The reason for this was that, although evidence shows that active mode users (walking, cycling), generally have higher reported subjective well-being (a component of this study's 'SE') by recall [9, 11, 12], this study could not take the risk of real 'other' travellers entering real-time data that countered this. This was because this study was part of a feasibility project to investigate whether other active transport users' higher SE can have an influence on car users. The others' active travel messages were skewed towards the positive and the 
others' car messages skewed towards the negative $(70 \%$ of the messages over the course of the study in each case) to give control over the experimental condition. There was no literature to support these chosen percentages but given our aim to promote changes in opinions and intentions to change, and acknowledging that many car users may report positive experiences of car use we made this choice.

As stated, the precise content of the messages was designed based on messages produced in a previous diary study by the researchers, where 30 participants recorded $\mathrm{SE}$ in written form for the commute over a one week period, without restriction (all transport modes included in this study). The team gathered all these messages and using a behavioural change theory, Socio-Cognitive Theory (SCT) [2] categorised them into 3 main components of the SCT: self-efficacy, outcome expectations and socio-structural impediments and facilitators (e.g. weather). To minimise the risk that participants would not believe that the feedback from others was not collected at real time (i.e. were 'designed' by the team), the following measures were taken: (i) recruitment adverts stated that the study was for drivers, walkers and cyclists but that this phase was recruiting drivers only; (ii) the feedback of messages from others were sent around typical commute times; (iii) the messages were designed to be relevant to the area of the study (road names, etc.); (iv) the planned messages were regularly checked against the weather forecast to ensure a match .

\subsection{Participants}

All participants were drivers who commuted to Loughborough (population 59,000) on a minimum of 3 days per week, typically with single occupancy. As individuals they were physically able to walk or cycle (i.e. no mobility problems). The maximum commuting distance (including drop-offs such as at schools) was 10miles (1 participant exceeded this by 1 mile).

Participants ( $\mathrm{n}=18$ ) were randomly split in two groups (control/test condition). A briefing session was used to ensure successful download of the app and to explain to participants what they required to do. All participants were told that the researchers were interested in gathering commuters' experiences. Information provided to the experimental group stated that the presence of others' SE was there for them to look at if they wished, in order that they were not primed to think this was a significant part (and purpose) of the study. After a briefing, the participants used the app for 2 weeks and then attended a session which comprised a questionnaire and a semistructured interview. The participant demographics were:

- Control group: Age 22 to 48 years, mean 36 years ( $S D=11.86) ; 1$ male; 8 females; Commuting distance 1 to 11 miles, mean 3.76 ( $S D=3.38$ )

- Experimental group: Age 27 to 56 years, mean 42 years $(\mathrm{SD}=10.15) ; 1$ male; 8 females; Commuting distance 1.5 to 9 miles, mean $4.56(\mathrm{SD}=2.58)$ 


\section{$3 \quad$ Results}

Data collected through the trial of the app, pre- and post- intervention questionnaires, semi-structured interviews and server stats (the latter only for the study screens; original app screen views could not be measured) were used within the analysis process. The semi-structured interviews were conducted individually with all 18 participants. Within the interviews various topics were explored including, intentions to change in the future; barriers to changing modes of transport, participants own subjective experiences (wellbeing and outcomes) and, for the experimental condition only, others' subjective experiences commuting to and from work. In addition it provided the opportunity to gain valuable feedback on the features of the intervention app and interaction with it. A thematic analysis was conducted on the qualitative data collected.

\subsection{Interaction with the app (study screens only)}

During the 2 week trial of the app, the participants in the control condition submitted on average 19.67 comments and those in the experimental condition submitted on average 18.67 comments (for the 2 week study, 2 commutes per day, the maximum possible was 20). Table 1 gives an overview of the number of times the screens designed for this study were viewed by participants. The views of the 'Capture SE' screen are high because all other screens were accessed via this careen.

Table 1. Mean screen views of the study screens over the 2-week trial

\begin{tabular}{|l|c|c|c|}
\cline { 2 - 4 } \multicolumn{1}{c|}{} & \multicolumn{3}{c|}{ Screen } \\
\cline { 2 - 4 } \multicolumn{1}{c|}{} & Capture SE & Own Comments & Others' Comments \\
\hline Control & 64.44 & 4.56 & n/a \\
\hline Experimental & 71.11 & 3.78 & 22.44 \\
\hline
\end{tabular}

\subsection{Experiences associated with interacting with the app}

By entering their own subjective experience ratings and comments, participants in both groups described that, as a result, they felt that they reflected on their own journeys more than usual, E8 (female, 27 years old) stated "It just kind of makes you think back about exactly what happened, how you felt while you were driving, how you felt while you were in the car." similarly C14 (female, 24 years old) stated "I sit there and try to think, what can I say today that doesn't involve talking about the traffic, which is the greatest influence on my journey enjoyment”.

In the experimental condition participants indicated their interest and often enjoyment in reading other users' comments; many participants expressed a particular interest in the active commuters' comments as illustrated by E15 (female, 30 years old) who stated "It was interesting to see the comments... especially not just the car users, but cyclists because sometimes... cyclists might cycle a bit dangerously and cut you up, but it's also interesting to see their perspective”. E11 (female, 37 years old) also expressed a similar view stating "Yeah, I did always read the ones on here. As a car 
driver, it makes you conscious of you know other people and their experiences and how things that you do can affect them." In addition to reflecting on their own commute, E11 also drew upon the prospect of changing modes of transport, stating "It's not a long walk for me, I have done it, I did used to cycle and obviously it ... it isn't stressful, it takes a bit more preparation in the morning.".

The 'real-time' aspect of the app was positively perceived by participants E13 (female,36) stated "I quite liked the different people's comments on how they got to work ... It was just quite interesting to see what other people were doing at the same time.” Whilst, E2 (male, 46 years old) was interested in how other commuters were coping with real-time weather conditions, noting "I always had a flick, especially when the weather's been bad; see what other people have thought!"

Participants discussed their intention to change in the future, many expressed barriers to change to more active modes for their commute, reasons included perceiving their commute distance was too long for alternative modes, having children to dropoff en route and safety concerns in regards to cycling. Some expressed an intention to change when the weather was warmer (bearing in mind this study was conducted in the late autumn/early winter), E4 (female, 40 years old) stated "Probably in the summer because then I can walk with my daughter halfway, drop her off at school and then I can walk to work.". Similarly C9 (female, 55 years old) stated "I would like to walk more rather than drive but it, again its weather dependent.”

Participants rated their intention to change before and after the study using a 5point scale (where weak intention $=1$, and strong intention $=5$ ), the mean scores before the intervention for the control and experimental conditions were $1.56(\mathrm{SD}=0.73)$ and $2.11(\mathrm{SD}=1.05)$, and after $2(\mathrm{SD}=1.12)$ and $2(\mathrm{SD}=1)$ respectively. A Wilcoxon test showed no significant difference between the pre-intervention intention to change scores and post-intervention intention to change score, in either the control or experimental condition (control condition: $\mathrm{Z}=-1.13, \mathrm{p}=0.26$; experimental condition: $\mathrm{Z}=-$ $0.58, \mathrm{p}=0.56$ ).

Other users' comments were accepted to be collected real time and directly from real people taking part in the study by a majority of participants in the experimental condition (6 out of 9). E12 (female, 48 years old) stated "No, no I just thought it was fun to do, I thought, oh all these people are having such a nice time, why am I so grumpy in the car!”. Of the other 3 , only 1 raised this prior to being told that they were not real-time and designed by the research team based on previous research, the other 2 only stated that they'd guessed this after being told.

\subsection{Usability of interface Design}

The usability of the interface would obviously be a factor in the level of use of the system, so this was also explored. During the semi-structured interviews participants indicated on a 7-point Likert scale (where strongly disagree $=1$ and strongly agree $=7$ ) how informative and easy to use they found each screen of the app (Figure 6). 


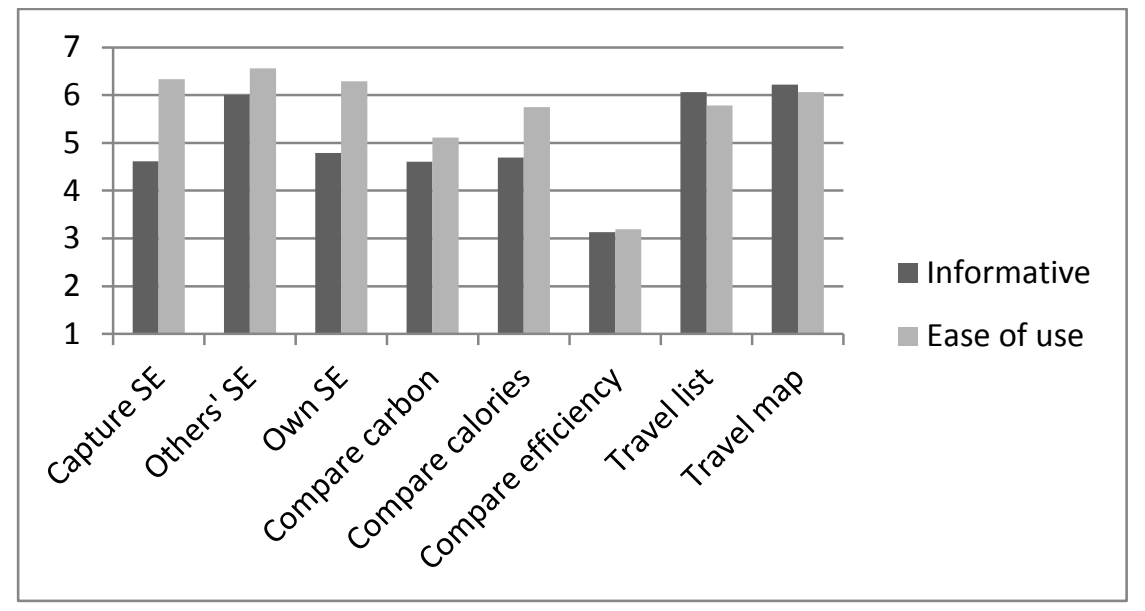

Fig. 6. Ratings of informativeness and ease of use for each screen

Participants reported a majority of the screens were easy to use, as illustrated by Figure 6. In particular the main 'intervention’ screens - Capture SE, Others SE (experimental group only) and Own SE - were considered to be easy to use, scoring above 6 on average, as did the Travel Map screen.

The three screens identified as most informative were Others' SE (accessible to the experimental group), Travel List and Travel Map. Frequently participants expressed a lack of understanding of the compare efficiency screen which scored the lowest in both measures, participants expressed a lack of understanding of the word 'efficiency' in the context it is used within the app. However it is important to bear in mind that the app developers did not intend for this particular screen to be for reflection purposes when it was devised for the originally intended business use. It is also worth noting participants estimated level of views for the compare efficiency screen was also the lowest across both conditions.

The app icon was automatically displayed in the notification bar of the phone, showing information on users' carbon consumption in the last 7 days; a majority of participants accessed the app through this option, some also noted the ease of access played a role in prompting the completion of the SE ratings and comments after each commute as E8 (female, 27 years old) stated "It was quite easy to remember to do it because the symbol was always there.”.

All participants across both conditions were given the option to keep or uninstall the app after the trial. Out of the 18 participants, 9 participants chose to keep the app installed on their phone, 6 from the experimental group and 3 from the control group.

\section{$4 \quad$ Discussion and conclusions}

The proposition being tested in this study was that reflecting on the more positive experiences of active transport mode users would cause a shift in car driver opinions, intentions or behaviours. The quantitative ratings of intention to change did not show 
a statistically significant difference before/after the study but we have to interpret this cautiously has we are not powered to detect differences. In general, the data collected reveals acceptability and feasibility of this intervention tool. The qualitative data from the interviews indicate several influences on opinions, and also intentions to change. Many of the participants stated that they enjoyed seeing the positive experiences of active travellers and made sure to look at these every day. From this it seems that the use of others' subjective experience shows promise as a component of behaviour change interventions. The intention to change ratings were given for the coming month and weather was a commonly mentioned barrier to change in the qualitative data (as well as distance, child drop-offs and cycling safety).

An unintended side-effect of feeding back others' SE was participants' reflection on how car drivers affect cyclists, and how the app started to give them an understanding of the cyclists' perspective. Although not a topic of investigation in this study, this aspect could indicate the value of this type of reflection for other transport challenges such as cyclist safety.

The design of the study app screens supported ease of use and therefore did not affect the impact of the 'SE reflection' intervention. Equally the usability of the original app screens was rated highly (with the exception of the 'Compare: Efficiency' screen). The usability of the study screens was further supported by the volume of 'Own SE' data collected which was close to the maximum of 20 (10 working days times 2 commutes). Others' SE was seen as more informative that 'Own SE' which probably explains why two thirds of the experimental group chose to keep the app after the trial (compared with one third of the control group).

In reflecting on the methodology, one of the most challenging aspects was to maintain believability of the others' real time collection of SE. The Method section explains how this was maximised and the results indicate that for the vast majority of participants the messages were perceived as real time.

In conclusion, as a first study into the use of reflection on others' SE as a behavioural intervention technique, this study shows that this approach has potential. Certainly, the participants' verbal reflection showed that it did make them contemplate more active/sustainable modes. Suggested further research includes: a study with larger numbers; a longer intervention period; the impact of repeating the study in more clement seasons; the combination with other behavioural change techniques shown to be effective in transport such as goal-setting and action-planning [14].

\section{Acknowledgements}

This study was carried out as part of the REFLECT project funded by the UK Engineering and Physical Sciences Research Council (EPSRC). The study was conducted by Loughborough University with significant support from Newcastle University. Thanks is also given to Jennifer Roberts of Sheffield University for her input. Loughborough University would also like to thank their collaborators TravelAi for providing the original app 'CarbonDiem' and their spirit of openness in allowing the sharing of results in this paper. 


\section{References}

1. ANABLE, J. AND GATERSLEBEN, B. 2005. All work and no play? The role of instrumental and affective factors in work and leisure journeys by different travel modes. Transportation Research Part A: Policy and Practice, 39, 163-181.

2. BANDURA, A. 2001. Social cognitive theory: An agentic perspective. Annual Review of Psychology 52, 1-26.

3. FANNING, J., MULLEN, SP. AND MCAULEY, E. 2012. Increasing Physical Activity With Mobile Devices: A Meta-Analysis, Journal of Medical Internet Research, 14(6):e161.

4. DE VOS, J., SCHWANEN, T., VAN ACKER, V. AND WITLOX, F. 2013. Travel and subjective well-being: a focus on findings, methods and future research needs. Transport Reviews 33, 421-442.

5. EPSTEIN, D.A., CORDEIRO, F., BALES, E., FOGARTY, J. AND MUNSON, S.A. Taming Data Complexity in Lifelogs: Exploring Visual Cuts of Personal Informatics Data.

6. ETTEMA, D., GÄRLING, T., ERIKSSON, L., FRIMAN, M., OLSSON, L.E. AND FUJII, S. 2011. Satisfaction with travel and subjective well-being: Development and test of a measurement tool. Transportation Research Part F: Traffic Psychology and Behaviour 14, 167-175.

7. FOGG, B.J. 2003. Persuasive Computer: Using Technology to Change What We Think and Do. Morgan Kaufman: SF, CA, USA.

8. JARIYASUNANT, J., ABOU-ZEID, M., CARREL, A., EKAMBARAM, V., GAKER, D., SENGUPTA, R. \& WALKER, J.L. 2013. Quantified Traveler: Travel Feedback Meets the Cloud to Change Behavior. Journal of Intelligent Transportation Systems: Technology, Planning, and Operations.

9. MORRIS, E.A. AND GUERRA, E. 2014. Mood and mode: does how we travel affect how we feel? Transportation 1-19.

10. OINAS-KUKKONEN, H. 2013. A foundation for the study of behavior change support systems. Personal and ubiquitous computing 17, 1223-1235.

11. OLSSON, L.E., GÄRLING, T., ETTEMA, D., FRIMAN, M. AND FUJII, S. 2013. Happiness and Satisfaction with Work Commute. Social Indicators Research 111, 255-263.

12. PÁEZ, A. AND WHALEN, K. 2010. Enjoyment of commute: A comparison of different transportation modes. Transportation Research Part A: Policy and Practice 44, 537-549.

13. PROST, S., SCHRAMMEL, J., RÖDERER, K. AND TSCHELIGI, M. 2013. Contextualise! personalise! persuade!: a mobile HCI framework for behaviour change support systems. In Proceedings of the 15th international conference on Human-computer interaction with mobile devices and services, Munich, Germany, Anonymous ACM, New York, NY, USA, 510-515.

14. ARNOTT, B., REHAKOVA, L., SNIEHOTTA, F. F., ROBERTS, J. R., AND ARAUJOSOARES, V. 2014. Efficacy of behavioural interventions for transport behaviour change: Systematic review and meta-analysis. International Journal of Behavioural Nutrition and Physical Activity. 11:133. 